\title{
Current regulatory approaches for accessing potential COVID-19 therapies
}

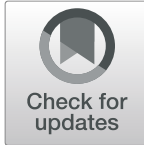

Vesa Halimi ${ }^{1}$, Armond Daci ${ }^{2}$, Simona Stojanovska ${ }^{3}$, Irina Panovska-Stavridis ${ }^{3}$, Milena Stevanovic ${ }^{4}$, Venko Filipce ${ }^{5}$ and Aleksandra Grozdanova ${ }^{1 *}$ (D)

\begin{abstract}
This commentary aims to elaborate challenges in the regulatory approaches for accessing and investigating COVID19 potential therapies either with off-label use, compassionate use, emergency use or for clinical trials. Since no therapies have been formally approved and completely effective and safe to date, the best clinical choice is acquired only after consistent and fair communication and collaboration between licensed clinicians, researchers, regulatory authorities, manufacturers and patients.
\end{abstract}

Keywords: COVID-19, Regulatory, EMA, FDA, Clinical studies, Compassionate use, Clinical practice, Off-label use, Emergency use

\section{Background}

The available scientific evidence and previous clinical experiences with SARS - CoV, MERS - CoV and even HIV have urged physicians to consider the use of an array of potential therapies like chloroquine and hydroxychloroquine, remdesivir, lopinavir/ritonavir, interferon beta, monoclonal antibodies, convalescent plasma, hyper immune globulin, antibody-rich blood products either alone or combined with supportive care (e.g., oxygenation, ventilation, fluid management) under several regulatory approaches that healthcare authorities made available. Nevertheless, the use of potential therapies in COVID-19 represents a critical responsibility, considering that these therapies are not approved by competent regulatory authorities to treat this disease, and respectively their safety and efficacy profile is under investigation [1].

\footnotetext{
* Correspondence: agrozdanova@ff.ukim.edu.mk

${ }^{1}$ Faculty of Pharmacy, University Ss. Cyril and Methodius, Skopje, North Macedonia

Full list of author information is available at the end of the article
}

Regulatory landscape for accessing COVID-19 therapies in the EU and US

There are several regulatory approaches for accessing potential therapies in COVID-19 and they can be classified as clinical trials, compassionate use, emergency use and off-label use (Table 1) [1-3].

The European Medicines Agency (EMA), even in this pandemic crisis, remained neutral by leaving within the remit of national regulatory authorities to launch their pragmatic regulatory pathways. Even though the EMA provided scientific advice for national regulatory agencies and manufacturers [3], many countries in Europe launched different regulatory approaches and protocols for accessing potential medicines [4-7]. Moreover, the dosing regimen in the protocols even for off-label use is not the same between countries, not to mention other programs. Under ideal conditions, the off-label program would constitute in the creation of a target patient population, informed consent and track and follow-up reports $[8,9]$. Still, prescribing an already approved medicine either for an indication, a dose or a way that is not approved for COVID-19 seems to be very challenging for clinicians. Therefore, under the COVID-19 emergency conditions, it is hard to believe that the off-label use 
Table 1 Regulatory attributes of clinical trials, compassionate use, emergency use and off-label use

\begin{tabular}{lllll}
\hline & Clinical trials & Compassionate use & Emergency use & Off-label use \\
\hline Regulatory approval & $\checkmark$ & $\checkmark$ & $\checkmark$ & No \\
Scope & Clinical research & Clinical practice & Clinical practice & Clinical practice \\
Informed consent & $\checkmark$ & $\checkmark$ & No & N/A a $^{\text {a }}$ \\
Target population & $\checkmark$ & $\checkmark$ & No \\
Safety reports & $\checkmark$ & $\checkmark$ & N/A a \\
Ethical board approval & $\checkmark$ & No & No \\
Control group & $\checkmark$ & No & No \\
Gathering evidence & Efficacy, Safety & Safety & Safety & N/A \\
Risk-benefit assessment & Group & Group & Group & On a case-by-case basis
\end{tabular}

${ }^{a}$ This table used unified regulatory characteristics of the EU and the US. Instead of "yes" we used the" tick symbol". Also, when a unified criteria was not met we used the not applicable (N/A) choice

would result to be the best approach for accessing potential medicines, considering the ongoing regulatory debates and the difficulties in assessing riskbenefit for each patient due to the pressurized and stressful situation [8,9].

Unlike EMA and some European countries, the Food and Drug Administration (FDA) was not very keen toward off-label use, by initiating the approval of the compassionate use, followed by the approval of emergency use for particular treatments and clinical trials [10]. Although terminology and modalities may not be identical, compassionate use programs demanding regulatory approval, informed consent and follow-up information are established in most countries $[11,12]$, and can be used to facilitate the access of seriously ill COVID-19 patients that cannot have access in clinical studies. Even though the compassionate use program is outlined within the framework of clinical practice, and does not have a control group, it can determine preliminarily safety and efficacy data until a level, within a well-formulated study design and hypothesis. Moreover, compassionate use might be seen as a treatment option for small countries which rarely have access to international clinical studies. In the past, during the interval of 5 years (19841989), the unapproved Ganciclovir was prescribed under the compassionate use for treatment of pneumonia Cytomegalovirus (CMV), retinitis CMV and colitis CMV for seriously ill immune-compromised patients and even today after 30 years it remains the preferred therapy for the treatment of CMV [13].

On the other hand, the idea for approving the emergency use relies not just in the emergency circumstances but also in providing legal protection for healthcare professionals and manufacturers for eventual adverse events and medication errors that the potential medicine may cause, as well as prescribing and dispensing a donated medicine free of charge within the framework of the hospital, and not obtaining informed consent for patients while tracking and reporting the treatment's outcomes $[14,15]$. Having regard to the fact that the manufacturer Gilead was called from the licensed clinicians to provide Remdesivir to hospitalized COVID-19 patients under the compassionate use, since 25th January 2020, and based on the methodological issues found at Grein et al.'s paper [16], it remains doubtful whether Gilead or regulatory authorities were not vulnerable toward this program. Even though the regulatory authorities usually approve most of the compassionate use requests when meeting the criteria, the key player in this program is the manufacturer, who is not legally obliged to provide the medicine to patients and is usually reluctant due to several factors (1) the outcomes of the compassionate program are not considered as an evidence for approval; (2) they may have limited quantities of the potential medicine already involved in clinical trials; (3) they may think they will be prejudiced if the medicine is proven to be ineffective and unsafe; (4) they want to bypass the regulatory requirements to provide followup information; (5) and the reimbursements issues in some countries [17-19]. According to regulatory authorities, the golden criterion for accessing and investigating potential medicines are clinical trials. The speed and volume of various clinical trials like Discovery, Recovery, Anger, Solidarity, New York Trial, characterized with different study designs, experimental arms, outcome measures, eligibility criteria and hypothesis emphasize the urge to produce significant safety and efficacy data in the middle of this pandemic crisis $[20,21]$. The golden criterion for the elucidation of the safety and efficacy profile of the potential therapies in COVID-19 does not rely just on the pursuit of randomized clinical studies under an ideal sample size and power, but one should analyze carefully and considerate the other aspects of the study design (e.g. study duration and masking), eligibility criteria (enrolling participants with similar characteristics) and clinically meaningful endpoints. 


\section{Conclusion}

Having regard to the diverse clinical presentation, course and progression of COVID-19 and the limited medical supply, regulatory authorities should establish robust regulatory programs that minimize clinical dilemmas in choosing the best therapy for COVID-19 patients. While clinicians should continue to inform COVID-19 patients or their legal representatives for the potential outcomes of therapies, obtaining informed consent from them is crucial for facilitating the clinical decision. Based on the available data, we believe that in COVID-19, no golden criterion is met in clinical research, not to mention clinical practice. Therefore, compassionate use program's mechanism with no control group might be shifted from "outside of clinical studies" to "parallel with clinical studies" to permit clinical studies to be implemented within their rigorous and narrow framework. However, how safe, effective and correct these current regulatory approaches have been, remains to be verified in the future.

\section{Abbreviations \\ EMA: European Medicines Agency; FDA: Food and Drug Administration; CMV: Cytomegalovirus; SARS - CoV-2: Severe Acute Respiratory Syndrome Coronavirus 2; SARS - CoV: Severe Acute Respiratory Syndrome Coronavirus: MERS - CoV: Middle East Respiratory Syndrome Coronavirus; HIV: Human Immunodeficiency Virus}

\section{Acknowledgements}

Vesa Halimi, Armond Daci, Simona Stojanovska, Irina Panovska-Stavridis, Milena Stevanovic, Venko Filipce, and Aleksandra Grozdanova, hereby declare that this section is not applicable.

\section{Authors' contributions}

Vesa Halimi, Armond Daci, Simona Stojanovska, Irina Panovska- Stavridis, Milena Stevanovic, Venko Filipce, and Aleksandra Grozdanova, hereby declare that $\mathrm{VH}$ conceived the idea, wrote and finalized the paper. AD, SN, IP, MS, VF, have contributed with their expertise, AG gave guidance, finalized and approved this commentary. The author(s) read and approved the final manuscript.

\section{Funding}

Vesa Halimi, Armond Daci, Simona Stojanovska, Irina Panovska- Stavridis, Milena Stevanovic, Venko Filipce, and Aleksandra Grozdanova, hereby declare that this option is not applicable.

\section{Availability of data and materials}

Vesa Halimi, Armond Daci, Simona Stojanovska, Irina Panovska- Stavridis, Milena Stevanovic, and Venko Filipce, Aleksandra Grozdanova, hereby declare that this option is not applicable.

\section{Ethics approval and consent to participate}

Vesa Halimi, Armond Daci, Simona Stojanovska, Irina Panovska- Stavridis, Milena Stevanovic, Venko Filipce and Aleksandra Grozdanova, hereby declare that this option is not applicable.

\section{Consent for publication}

Vesa Halimi, Armond Daci, Simona Stojanovska, Irina Panovska- Stavridis, Milena Stevanovic, Venko Filipce and Aleksandra Grozdanova, hereby declare that this option is not applicable.

\section{Competing interests}

Vesa Halimi, Armond Daci, Simona Stojanovska, Irina Panovska- Stavridis, Milena Stevanovic, Venko Filipce and Aleksandra Grozdanova, hereby declare that no competing interests exist.

\section{Author details}

${ }^{1}$ Faculty of Pharmacy, University Ss. Cyril and Methodius, Skopje, North Macedonia. ${ }^{2}$ Department of Pharmacy, Faculty of Medicine, University of Prishtina, Prishtina, Kosovo. ${ }^{3}$ University Clinic of Hematology, Medical Faculty, University Ss. Cyril and Methodius, Skopje, North Macedonia. ${ }^{4}$ University Clinic of Infection diseases and febrile conditions, Medical Faculty, University Ss. Cyril and Methodius, Skopje, North Macedonia. ${ }^{5}$ University Clinic for Neurosurgery, Medical Faculty, University Ss. Cyril and Methodius, Skopje, North Macedonia.

Received: 27 April 2020 Accepted: 1 May 2020

Published online: 16 May 2020

\section{References}

1. Kalil AC. Treating COVID-19 - Off-Label Drug Use, Compassionate Use, and Randomized Clinical Trials during Pandemics. JAMA - J Am Med Assoc. 2020.

2. Food and Drug Administration FDA. Emergency Use Authorization (EUA) information, and list of all current EUAs. Available from: https://www.fda. gov/emergency-preparedness-and-response/mcm-legal-regulatory-andpolicy-framework/emergency-use-authorization\#2019-ncov.

3. European Medicines Agency. Treatments and vaccines for COVID-19| European Medicines Agency. [Cited 2020 Apr 21]. Available from: https:// www.ema.europa.eu/en/human-regulatory/overview/public-health-threats/ coronavirus-disease-covid-19/treatments-vaccines-covid-19.

4. Italian Medicines Agency (AIFA). Emergenza COVID-19| Agenzia Italiana del Farmaco. [Cited 2020 Apr 21]. Available from: https://www.aifa.gov.it/ emergenza-covid-19.

5. Federal Institute for Drugs and Medical Devices. BfArM - Coronavirus SARSCoV-2. [Cited 2020 Apr 21]. Available from: https://www.bfarm.de/DE/ Service/Presse/Themendossiers/Coronavirus/_node.html.

6. The French National Agency for Medicines and Health Products Safety (ansm). Médicaments - Nos informations de sécurité, avis et recommandations face au COVID - 19 - ANSM : Agence nationale de sécurité du médicament et des produits de santé. [Cited 2020 Apr 24]. Available from: https://www.ansm.sante.fr/Dossiers/COVID-19/MedicamentsNos-informations-de-securite-avis-et-recommandations-face-au-COVID-19/ (offset)/1\#paragraph_64595.

7. Federal agency for medicines and health products (famph). Coronavirus: what medicinal products are used in the fight against COVID-19? | FAMHP. [Cited 2020 Apr 24]. Available from: https://www.famhp.be/en/news/ coronavirus_what_medicinal_products_are_used_in_the_fight_against_ covid_19.

8. França K, Litewka S. Controversies in off-label prescriptions in dermatology: the perspective of the patient, the physician, and the pharmaceutical companies. Int J Dermatol. 2019;58(7):788.

9. Lenk C, Duttge G. Ethical and legal framework and regulation for off-label use: European perspective. Ther Clin Risk Manag. 2014;10:537.

10. Food and Drug Administration (FDA). Coronavirus (COVID-19) Update: FDA Continues to Facilitate Development of Treatments | FDA. [Cited $2020 \mathrm{Apr}$ 24]. Available from: https://www.fda.gov/news-events/pressannouncements/coronavirus-covid-19-update-fda-continues-facilitatedevelopment-treatments.

11. Borysowski J, Górski A. Compassionate use of unauthorized drugs: legal regulations and ethical challenges. Eur J Intern Med. 2019;65:12.

12. Balasubramanian G, Morampudi S, Chhabra P, Gowda A, Zomorodi B. An overview of compassionate use programs in the european union member states. Intractable Rare Dis Res. 2016;5(4):244.

13. Buhles WC. Compassionate use: a story of ethics and science in the development of a new drug. Perspect Biol Med. 2011;54(3):304.

14. Food and Drug Administration (FDA). FDA COMBATING COVID-19 WITH THERAPEUTICS | FDA [Internet]. [cited 2020 Apr 21]. Available from: https:// www.fda.gov/media/136832/download.

15. The French National Agency for Medicines and Health Products Safety (ansm). Plaquenil et Kaletra : les traitements testés pour soigner les patients COVID-19 ne doivent être utilisés qu'à I'hôpital - Point d'information - ANSM : Agence nationale de sécurité du médicament et des produits de santé. [Cited 2020 Apr 24]. Available from: https://www.ansm.sante.fr/S-informer/ Points-d-information-Points-d-information/Plaquenil-et-Kaletra-lestraitements-testes-pour-soigner-les-patients-COVID-19-ne-doivent-etreutilises-qu-a-l-hopital-Point-d-information. 
16. Grein J, Ohmagari N, Shin D, Diaz G, Asperges E, Castagna A, et al. Compassionate use of Remdesivir for patients with severe Covid-19. N Engl J Med. 2020;1:1-10 Available from: http://www.ncbi.nIm.nih.gov/ pubmed/32275812.

17. Zettler PJ. Compassionate use of experimental therapies: who should decide? EMBO Mol Med. 2015;7(10):1248.

18. Jerome RN, Edwards TL, Boswell HC, Bernard GR, Harris PA, Pulley JM. Recommendations to facilitate expanded access to investigational therapies for seriously ill patients. Acad Med. 2016;91(3):305.

19. Mackey TK, Schoenfeld VJ. Going "social" to access experimental and potentially life-saving treatment: an assessment of the policy and online patient advocacy environment for expanded access. BMC Med. 2016;14:17.

20. Association AM. Pharmacologic Treatments for Coronavirus Disease 2019 (COVID-19): A Review, vol. 2019; 2020.

21. U.S. National Library of Medicine. COVID-19 Studies from the World Health Organization Database - ClinicalTrials.gov. [Cited 2020 Apr 21]. Available from: https://clinicaltrials.gov/ct2/who_table.

\section{Publisher's Note}

Springer Nature remains neutral with regard to jurisdictional claims in published maps and institutional affiliations.

Ready to submit your research? Choose BMC and benefit from:

- fast, convenient online submission

- thorough peer review by experienced researchers in your field

- rapid publication on acceptance

- support for research data, including large and complex data types

- gold Open Access which fosters wider collaboration and increased citations

- maximum visibility for your research: over $100 \mathrm{M}$ website views per year

At BMC, research is always in progress.

Learn more biomedcentral.com/submissions 